\title{
Crystallite Size Evaluation of ZnO Nanoparticles via Transmission Electron Microscopy and X-ray Powder Diffraction
}

Jonathan E. Cowen ${ }^{1}$, Ashley E. Harris ${ }^{2}$, Cecelia C. Pena ${ }^{2}$, Stephen C. Bryant ${ }^{2}$, Allison J. Christy $^{2}$, and Jerry D. Harris ${ }^{2 *}$

1. Swagelok Center for the Surface Analysis of Materials, Case Western Reserve University, Cleveland, OH, U.S.A.

2. Department of Chemistry, Northwest Nazarene University, Nampa, ID, U.S.A.

Zinc Oxide has received a considerable amount of attention over the past decades due to its unique electronic and optical properties. The material is readily available, inexpensive, and environmentally friendly compared to other semiconducting materials ${ }^{1}$. These properties make $\mathrm{ZnO}$ an excellent candidate material for applications such as: photovoltaic devices ${ }^{2}$, gas sensors ${ }^{3}$, transparent conductive oxides (TCO) ${ }^{4}$, biomedical devices ${ }^{5}$, and photocatalysis ${ }^{6}$ among many others. Zinc oxide was synthesized by alkali precipitation using aqueous solutions of zinc acetate, various amines, and precipitated with the addition of $\mathrm{NaOH}$. The resulting materials were characterized with Transmission Electron Microscopy (TEM), X-ray Diffraction (XRD), and the Brunauer-Emmett-Teller (BET) method. The optical band gap of the material was determined with Ultraviolet-visible reflectance spectroscopy.

While the BET method was used to determine the surface area of the resulting material, the main focus of this presentation is on the crystallite size determination from both XRD (shown in fig. 1) and TEM (shown in fig. 2-3). Williamson-Hall plots were generated to separate out the effect of size broadening from micro-strain broadening on the XRD peaks to better calculate the actual crystallite size. The results were then compared to TEM micrographs of the respective samples. The photochemical reactivity of the different materials was evaluated by using them to degrade the organic dye malachite green in aqueous solutions under UVA irradiation. In general, it was found that for a given morphology, smaller particles yielded faster decomposition rates than larger particles.

References:

[1] J. Iqbal, X. Liu, A. Majid, and R. Yu, Journal of Superconductivity and Novel Magnetism, vol. 24 (2011) p. 699

[2] A. Ennaoui, M. Weber, R. Scheer, H.J. Lewerenz, Sol. Energy Mater. Sol. Cells. Vol. 54 (1998) p. 277

[3] F. Paraguay D., M. Miki-Yoshida, J. Morales, J. Solis, W. Estrada L., Thin Solid Films vol. 373 (2000) p. 137

[4] R. Das, S. Ray, J. Phys. D: Appl. Phys. Vol. 36 (2003) p.152

[5] K.M. Reddy, K. Ferris, J. Bell, D.G. Wingett, C. Hanley, A. Punnoose, Appl. Phys. Lett vol. 90 (2007) p. 213902

[6] J.O. Saucedo-Lucero, S. Arriaga, J. of Chemical Engineering vol. 218 (2013) p. 358 


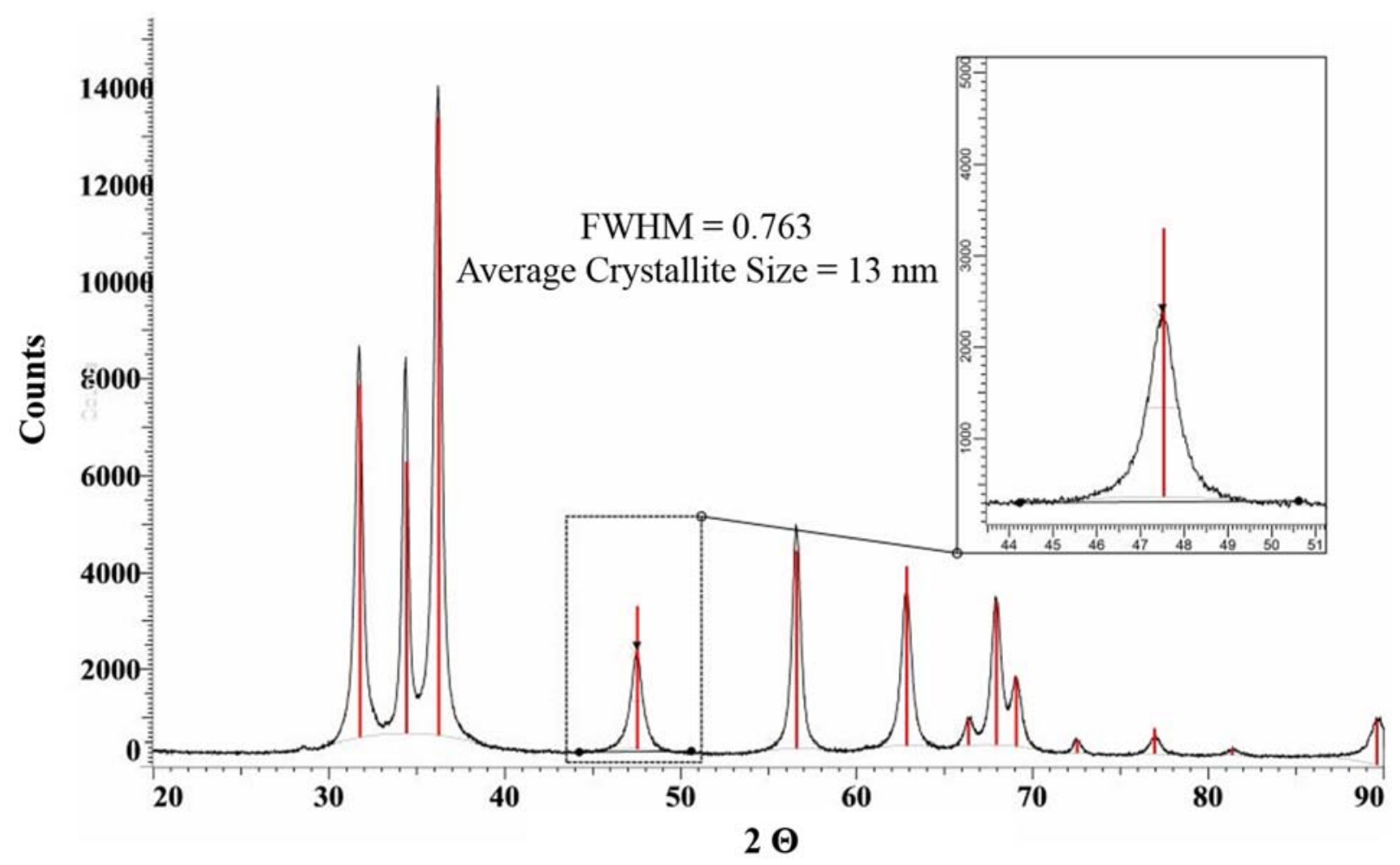

Figure 1. XRD Pattern of Zincite nanoparticles displaying the average crystallite size determined from the Scherrer Equation

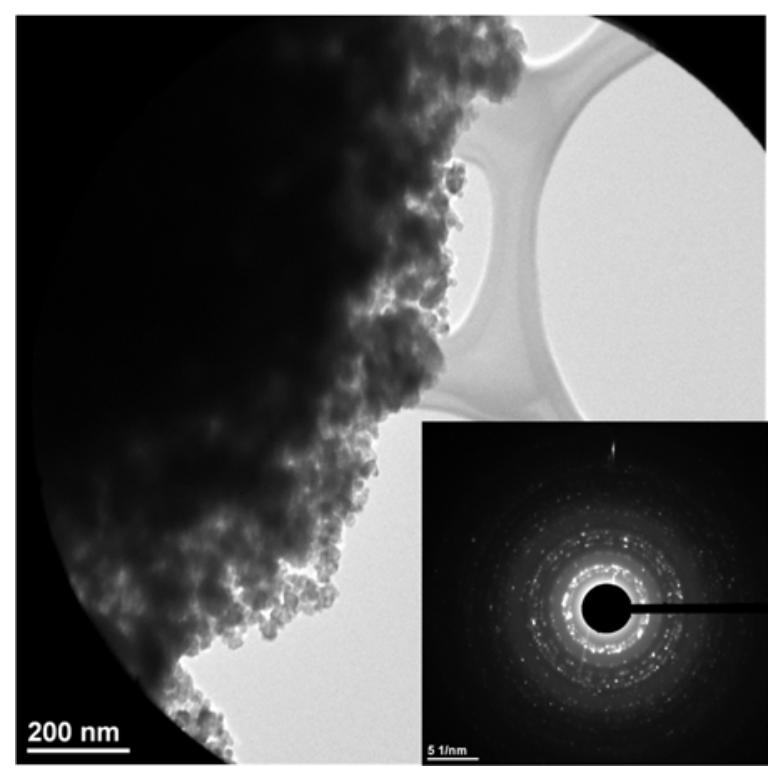

Figure 2. Zero loss bright field image of an agglomerate of Zincite nanoparticles with corresponding electron diffraction pattern.

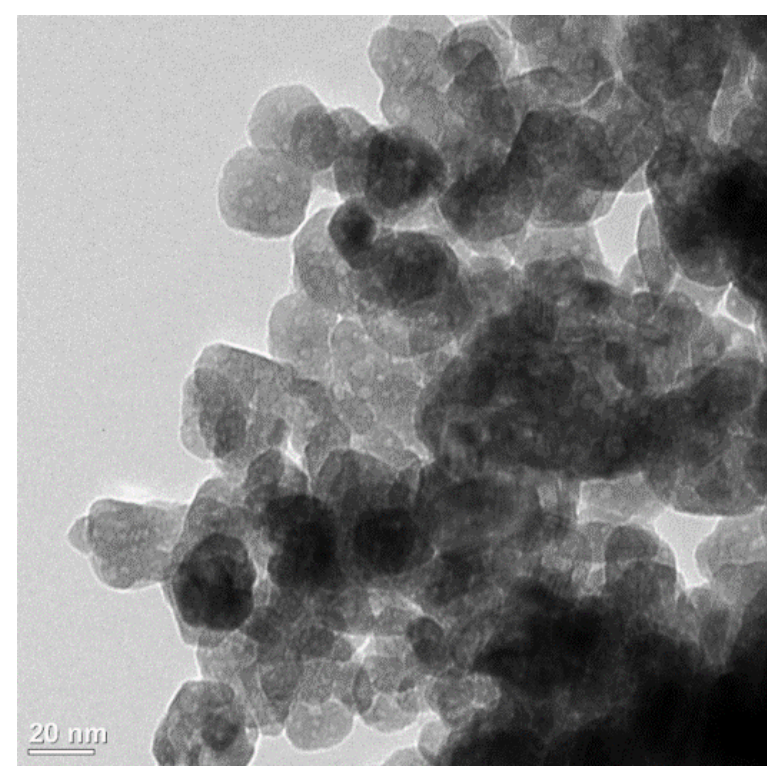

Figure 3. Zero loss bright field image of Zincite nanoparticles taken at 100,000X. 